\title{
Synthesis, Characterization and Evaluation of Biological Activities of Sn(II) Complexes of Schiff base Incorporating Sulpha Drugs
}

\author{
KIRAN MEENA ${ }^{1,2}$, VIRENDRA SINGH SHEKHAWAT ${ }^{2}$, SARITA VARSHNEY ${ }^{2}$ \\ and A. K. VARSHNEY2*
}

1Department of Chemistry, Mohanlal Sukhadia University, Udaipur-313001, Rajasthan, India. ${ }^{2}$ Department of Chemistry, University of Rajasthan, Jaipur-302004, India.

http://dx.doi.org/10.13005/ojc/370617

(Received: August 25, 2021; Accepted: November 18, 2021)

\begin{abstract}
In this study, we report synthesis, characterization and biological activities of four sulpha drug based Schiff base ligands and their Sn(II) complexes. The Schiff bases and their Sn(II) complexes have been synthesized by traditional methods and characterized by the spectral techniques IR, NMR $\left({ }^{1} \mathrm{H}\right.$ and $\left.{ }^{13} \mathrm{C}\right)$, mass and TGA-DTA. Newly synthesized Schiff bases $\left(\mathrm{L}^{1}-\mathrm{L}^{4}\right)$ and their $\mathrm{Sn}(\mathrm{II})$ complexes (C-1 to C-4) have been screened for antibacterial activity against bacterial strains $S$. aureus, S. pyogenus, E. coli, $P$. aeruginosa and antifungal activity against fungal strains $C$. albicans, $A$. niger, $A$. clavatus using broth micro dilution method. Best antimicrobial activity was shown by $\mathrm{C}-3$ complex against $E$. coli (MIC, $50.0 \mu \mathrm{g} / \mathrm{mL}$ ) and $A$. niger microbial strains (MIC, 100 $\mu \mathrm{g} / \mathrm{mL}$ ). Moreover, antimalarial activity against Plasmodium falciparum was also studied. Complex $\mathrm{C}-3$ was found to be more active against parasite $P$. falciparum $\left(\mathrm{IC}_{50}, 0.04 \mu \mathrm{g} / \mathrm{mL}\right)$. Results showed that dichloride tin complexes are more active with respect to their corresponding Schiff base ligands.
\end{abstract}

Keywords: Sulpha drug Schiff base, Sn(II) complexes, Antibacterial, Antifungal and Antimalarial activities.

\section{INTRODUCTION}

Hugo Schiff in 1864 first reported preparation and coordination properties of Schiff bases. Schiff bases which have azomethine linkage $(-\mathrm{CH}=\mathrm{N}-)$ can be synthesized by condensation of active carbonyl compounds with primary amines under acidic or basic conditions ${ }^{1-2}$. The azomethine linkage is responsible for coordination properties and biological activities such as antimalarial ${ }^{3}$, anticancer ${ }^{4}$, antibacterial $^{5}$, antifungal ${ }^{6}$, antitubercular ${ }^{7}$, anti- inflammatory ${ }^{8}$, antitumor ${ }^{9}$, insecticidal ${ }^{9}$, catalytic activity $^{10}$ and other applications ${ }^{11}$.

Bacterial and fungal infectious diseases increased mortality in living organism. Mainly following bacterial strains E. coli, P. aeruginosa, $S$. aureus, $S$. pyogenus are responsible for many diseases like food borne diseases and food poisoning etc. With the time microbes may have developed resistance to antibiotics that causes many difficulties in treatment of such diseases and it has

This is an Open Access article licensed under a Creative Commons license: Attribution 4.0 International (CC- BY). Published by Oriental Scientific Publishing Company @ 2018

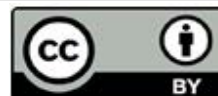


created global concern to human health. Thus, it is urgent necessity to the exploration and development of new more effective antibacterial and antifungal drugs. Schiff base and their complexes has potential to be good antibacterial and antifungal agents ${ }^{12-13}$.

Malaria is one of the most destructive infectious diseases and causing serious health problems. $P$. falciparum, $P$. ovale, $P$. vivax and $P$. malaria ${ }^{14}$ species of plasmodium are responsible for malaria in humans. Among these species $P$. falciparum parasite is most dangerous and which is vectored by female anopheles mosquito. For the treatment of malaria, first discovered antimalarial drug was quinine, isolated from the bark of cinchona plant ${ }^{15}$. Currently, chloroquine is used to treat malaria because it is less toxic, cheaper and effective drug ${ }^{16}$.<smiles>CCN(C)CCCC(C)Nc1ccnc2cc(Cl)ccc12</smiles>

Fig. 1. Structure of chloroquine (CQ)

The sulpha drugs containing $\left(-\mathrm{SO}_{2} \mathrm{NH}-\right)$ moiety are extensively used as, antimicrobial ${ }^{17}$, anti-inflammatory ${ }^{18}$, antibacterial ${ }^{19}$, antifungal ${ }^{19}$, anticancer ${ }^{20}$, antiviral agents ${ }^{20}$, diuretics, anticonvulsants, hypoglycaemic, HIV protease inhibitors activity and helpful in both prevention and cure of bacterial infections ${ }^{21-27}$. Sn(II) compounds have many applications in polymer and dyes industry as catalyst, antitumor and herbicidal use, as photostabilizers, chelating ability, thermal stability, optical nonlinearity ${ }^{28}$, miticides, molluscides, fungicides, surface disinfectants, wood preservatives $^{29}$ and marine antifouling ${ }^{30}$ paints.

Methyltetrahydrofuran (MeTHF) is an environmently friendly solvent. It has boiling point $80^{\circ} \mathrm{C}$ which is higher than boiling point of tetrahydrofurane (THF) $66^{\circ} \mathrm{C}$ so it increases reaction rate. However, THF is highly miscible with water and generate considerable waste water and waste solvent while MeTHF is less soluble in water, it is low peroxide producing green solvent and it can be easily dried ${ }^{31}$.

Here in, we describe synthesis, characterization and biological evaluation of various types of sulpha drugs based Schiff base ligands and their Sn(II) complexes using MeTHF as green solvent.

\section{EXPERIMENTAL}

\section{MATERIALS AND METHODS}

Anisaldehyde, sulphacetamide, sulphamethazine and sulphamerazine were bought from Sigma-Aldrich, India. Sulphadiazine and Anhydrous metal salt (tin chloride) were purchased from Alfa-Aesar. Standard method ${ }^{32}$ was used for drying the solvents. All the reactions were carried out under anhydrous conditions. Purification of all the compounds has been checked by aluminium silica plate. Melting points of were measured by the Myra melting point apparatus. IR spectrum was recorded using (Bruker alpha-T FT-IR spectrometer) from Department of Chemistry, MLSU, Udaipur (Raj.), India. Spectrum of ${ }^{1} \mathrm{H} \&{ }^{13} \mathrm{C}-\mathrm{NMR}$ were recorded (BRUKER AVANCE NEO $500 \mathrm{MHz}$ NMR SPECTROMETER) using DMSO-d ${ }_{6}$ solution from SAIF, Panjab University Chandigarh, India. Mass spectrum was obtained (SYNAPT-XSDBATOF-MS ES+ mass spectrometer) from SAIF, Chandigarh, India. Thermogravimetric analysis was recorded (DTA-TGA Perkin Elmer 6000) from MNIT Jaipur, India. Antimicrobial activities of the synthesized compounds have been performed at Micro Care Laboratory, Surat (Gujarat), India.

\section{Synthesis of ligands}

Sulpha drug based Schiff base ligands have been synthesized as per reported method ${ }^{33-34}$. In this method, we have used a $100 \mathrm{~mL}$ neat and clean round bottom flask, $5 \mathrm{~mL}$ of alcoholic solution of anisaldehyde $(1.22 \mathrm{~mL}, 0.01 \mathrm{~mol})$ was mixed with hot absolute ethanol-acetone solution of sulphacetamide (1.24 g, $0.01 \mathrm{~mol})$ / sulphadiazine (2.50 g, 0.01mol)/ sulphamethazine $(2.7 \mathrm{~g}, 0.01 \mathrm{~mol}) /$ sulphamerazine $(2.64 \mathrm{~g}, 0.01 \mathrm{~mol})$ in $1: 1 \mathrm{molar}$ ratio. To this solution we have added 4-5 drops of catalyst (glacial acetic acid) and refluxed for about $24 \mathrm{~h}$ on oil bath at $60-70^{\circ} \mathrm{C}$ temperature. Completion of the reaction has been checked by TLC and then it was allowed to cool in refrigerator. The obtained precipitates were filtered off, washed several times with the cold ethanol and purification was done by recrystallization from absolute ethanol. The scheme of the reaction is shown in Figure 2. 
<smiles>COc1ccc(C=O)cc1</smiles><smiles>[R]NS(=O)(=O)c1ccc(N)cc1</smiles>

$\mathrm{HO}$

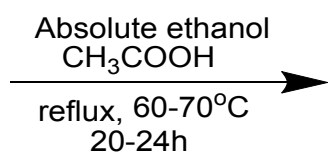

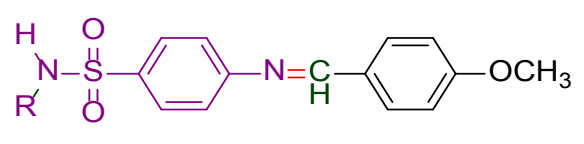

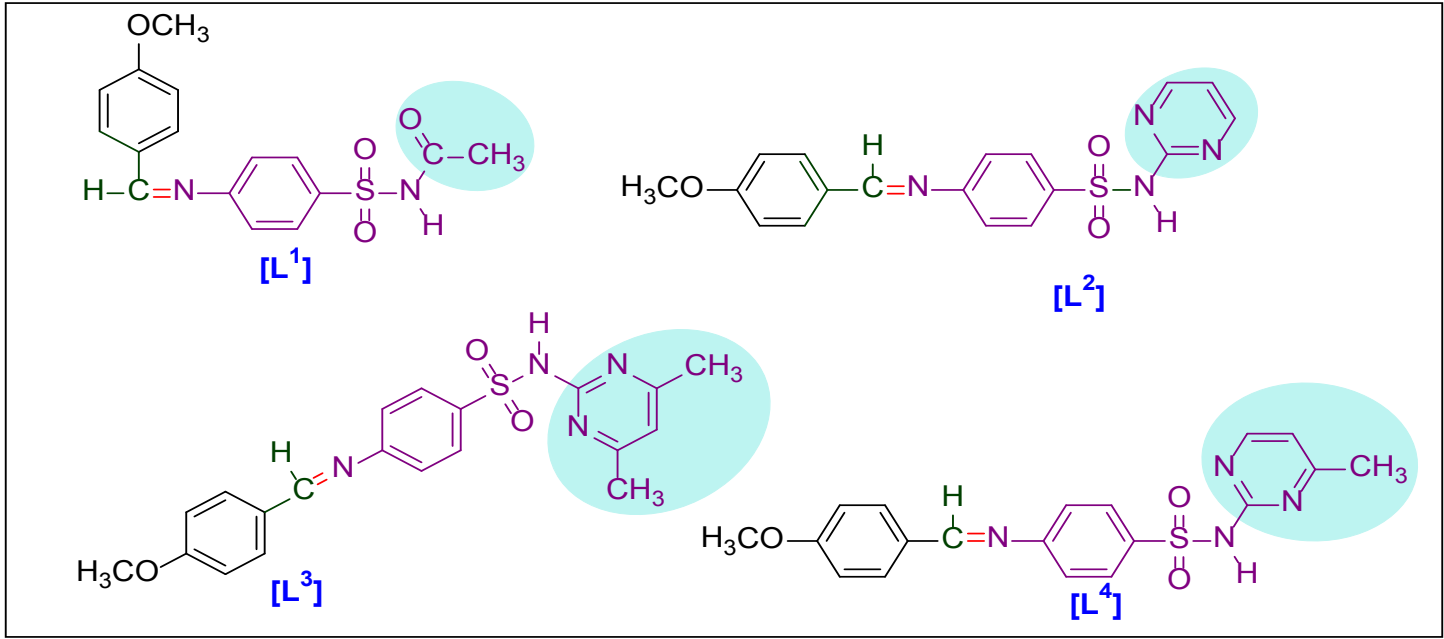

Fig. 2. Synthesis of sulphadrug based Schiff base ligands $\left[L^{1}-L^{4}\right]$

$\mathrm{N}$-((4-((4-methoxybenzylidene) amino) phenyl) sulfonyl)acetamide $\left[\mathrm{L}^{1}\right]$

$\mathrm{C}_{16} \mathrm{H}_{16} \mathrm{~N}_{2} \mathrm{O}_{4} \mathrm{~S}$; Yellow colored; yield, 68.42\%; m.p. $210^{\circ} \mathrm{C}$; FT-IR $\left(\mathrm{KBr}, \vee \mathrm{cm}^{-1}\right)$ : $\vee \mathrm{C}=\mathrm{N}\left(1635 \mathrm{~cm}^{-1}\right)$, v $\mathrm{NH}\left(3260 \mathrm{~cm}^{-1}\right), \vee \mathrm{OCH}_{3}\left(2920 \mathrm{~cm}^{-1}\right), \vee \mathrm{COCH}_{3}(1700-$ $\left.1720 \mathrm{~cm}^{-1}\right), v \mathrm{SO}_{2} 1315 \& 1150 \mathrm{~cm}^{-1}$ (Asy.\& Sym.), $v$ $\mathrm{C}=\mathrm{C}_{\text {aro }}\left(1495 \mathrm{~cm}^{-1}\right), \mathrm{v}=\mathrm{C}-\mathrm{H}\left(3050 \mathrm{~cm}^{-1}\right) .{ }^{1} \mathrm{H}-\mathrm{NMR}: \delta$ $(p p m)=8.55(\mathrm{~S}, 1 \mathrm{H}, \mathrm{CH}=\mathrm{N}), 11.67(\mathrm{~S}, 1 \mathrm{H}, \mathrm{NH}), 1.87$ $\left(\mathrm{S}, 3 \mathrm{H}, \mathrm{CH}_{3}\right), 3.85\left(\mathrm{~S}, 3 \mathrm{H}, \mathrm{OCH}_{3}\right), 7.10(\mathrm{~d}, 2 \mathrm{H}, \mathrm{J}=1.8$ $\mathrm{Hz}$ ), 7.87 (dd, 2H, J=2.0 Hz), 7.38 (d, 2H, J=1.75 $\mathrm{Hz}), 6.61(\mathrm{~d}, 2 \mathrm{H}, \mathrm{J}=1.75 \mathrm{~Hz}) .{ }^{13} \mathrm{C}-\mathrm{NMR}: \delta(\mathrm{ppm})$ $=162.38(-\mathrm{CH}=\mathrm{N}-), 23.07\left(\mathrm{CH}_{3}\right), 55.49\left(\mathrm{OCH}_{3}\right)$, $191.21(\mathrm{C}=\mathrm{O}), 135.47\left(\mathrm{C}_{1}\right), 121.19\left(\mathrm{C}_{2}, \mathrm{C}_{6}\right), 123.73$ $\left(\mathrm{C}_{3}, \mathrm{C}_{5}\right), 142.00\left(\mathrm{C}_{4}\right), 128.32\left(\mathrm{C}_{7}\right), 130.92\left(\mathrm{C}_{8}, \mathrm{C}_{12}\right)$, $114.42\left(\mathrm{C}_{9}, \mathrm{C}_{11}\right), 164.12\left(\mathrm{C}_{10}\right)(\mathrm{Ar}-\mathrm{C})$. Mass $(\mathrm{m} / \mathrm{z})$ : Base peak $=332.62(\mathrm{M})^{+}\left[\mathrm{C}_{16} \mathrm{H}_{16} \mathrm{~N}_{2} \mathrm{O}_{4} \mathrm{~S}\right]^{+}, 333.67$ $(\mathrm{M}+1)\left[\mathrm{C}_{16} \mathrm{H}_{17} \mathrm{~N}_{2} \mathrm{O}_{4} \mathrm{~S}\right]+, 107.95\left[\mathrm{SO}_{2} \mathrm{COCH}_{3}\right]^{+}$,cal. 332. 08 ; found 332.62 .

\section{4-((4-methoxybenzylidene) amino)- $\mathrm{N}$-(pyrimidin-} 2-yl) benzenesulfonamide [ $\left.\mathrm{L}^{2}\right]$

$\mathrm{C}_{18} \mathrm{H}_{16} \mathrm{~N}_{4} \mathrm{O}_{3} \mathrm{~S}$; Dark red colored; yield, $50 \%$; m.p. $210-240^{\circ} \mathrm{C}$; FT-IR $\left(\mathrm{KBr}, \mathrm{v} \mathrm{cm}^{-1}\right)$ : $\vee \mathrm{CH}=\mathrm{N}(1620$ $\left.\mathrm{cm}^{-1}\right), \vee \mathrm{NH}\left(3270 \mathrm{~cm}^{-1}\right), \vee \mathrm{SO}_{2}\left(1320 \& 1200 \mathrm{~cm}^{-1}\right)$, $\vee \mathrm{C}=\mathrm{C}_{\text {aro }}\left(1572 \mathrm{~cm}^{-1}\right), \mathrm{V}=\mathrm{C}-\mathrm{H}\left(2970 \mathrm{~cm}^{-1}\right) .{ }^{1} \mathrm{H}-\mathrm{NMR}$ : $\delta(\mathrm{ppm})=8.52(\mathrm{~S}, 1 \mathrm{H}, \mathrm{CH}=\mathrm{N}), 11.66(\mathrm{~S}, 1 \mathrm{H}, \mathrm{NH})$, $3.65\left(\mathrm{~S}, 3 \mathrm{H},-\mathrm{OCH}_{3}\right), 6.73(\mathrm{~d}, 2 \mathrm{H}, \mathrm{J}=9.05 \mathrm{~Hz}), 6.67$ (dd, $2 \mathrm{H}, \mathrm{J}=10 \mathrm{~Hz}), 8.47$ (d, 2H, J= $4.85 \mathrm{~Hz}$ ), 7.66 (d, $2 \mathrm{H}, \mathrm{J}=4.85 \mathrm{~Hz}$ ), 8.52 (d, 2H, J= $4.95 \mathrm{~Hz}, \mathrm{H}_{\text {diazine }}$ ), $8.27\left(\mathrm{t}, 1 \mathrm{H}, \mathrm{J}=5 \mathrm{~Hz}, \mathrm{H}_{\text {diazine }}\right)$. ${ }^{13} \mathrm{C}-\mathrm{NMR}: \delta(\mathrm{ppm})=$ $158.14(\mathrm{CH}=\mathrm{N}), 55.93\left(\mathrm{OCH}_{3}\right), 115.59\left(\mathrm{C}_{1}\right), 122.40$ $\left(\mathrm{C}_{2}, \mathrm{C}_{4}\right), 161.06\left(\mathrm{C}_{3}\right), 142.99\left(\mathrm{C}_{5}\right), 131.46\left(\mathrm{C}_{6}, \mathrm{C}_{10}\right)$, $130.19\left(\mathrm{C}_{7}, \mathrm{C}_{9}\right), 150.99\left(\mathrm{C}_{8}\right), 128.40\left(\mathrm{C}_{11}\right), 130.20\left(\mathrm{C}_{12}\right.$, $\left.\mathrm{C}_{16}\right), 114.40\left(\mathrm{C}_{13}, \mathrm{C}_{15}\right), 162.90\left(\mathrm{C}_{14}\right)(\operatorname{Ar}-\mathrm{C})$. Mass $(\mathrm{m} / \mathrm{z})$ : $368.60(\mathrm{M})+\left[\mathrm{C}_{18} \mathrm{H}_{16} \mathrm{~N}_{4} \mathrm{O}_{3} \mathrm{~S}\right]^{+}, 370.56(\mathrm{M}+2), 372.56$ $(\mathrm{M}+4), 374.58(\mathrm{M}+6), 376.60(\mathrm{M}+8), 378.57(\mathrm{M}+10)$, $158.88\left[\mathrm{C}_{4} \mathrm{H}_{4} \mathrm{~N}_{3} \mathrm{SO}_{2}\right]^{+}$, Cal. 368.09; found 368.41.

\section{N-(4,6-dimethylpyrimidin-2-yl)-4-((4-methoxy} benzylidene) amino) benzenesulfonamide $\left[\mathrm{L}^{3}\right]$

$\mathrm{C}_{20} \mathrm{H}_{20} \mathrm{~N}_{4} \mathrm{O}_{3} \mathrm{~S}$; Orange colored; yield, 79\%; m.p. $190^{\circ} \mathrm{C}$; FT-IR $\left(\mathrm{KBr}, \vee \mathrm{cm}^{-1}\right): \vee \mathrm{CH}=\mathrm{N}(1626$ $\left.\mathrm{cm}^{-1}\right), \vee \mathrm{NH}\left(3350 \mathrm{~cm}^{-1}\right), \vee \mathrm{CH}_{3}\left(2855 \mathrm{~cm}^{-1}\right), \vee \mathrm{OCH}_{3}$ $\left(2950 \mathrm{~cm}^{-1}\right), \vee \mathrm{SO}_{2}\left(1350 \& 1247 \mathrm{~cm}^{-1}\right) .{ }^{1} \mathrm{H}-\mathrm{NMR}: \delta$ $(\mathrm{ppm})=8.52(\mathrm{~S}, 1 \mathrm{H}, \mathrm{CH}=\mathrm{N}), 11.50(\mathrm{~S}, 1 \mathrm{H}, \mathrm{NH}), 3.81$ $\left(\mathrm{S}, 3 \mathrm{H}, \mathrm{OCH}_{3}\right), 7.07$ (d, 2H, J=2.7 Hz), 7.02 (dd, $2 \mathrm{H}$, $\mathrm{J}=5 \mathrm{~Hz}), 6.78(\mathrm{~d}, 2 \mathrm{H}, \mathrm{J}=8.5 \mathrm{~Hz}), 7.87(\mathrm{~d}, 2 \mathrm{H}, \mathrm{J}=$ $8.5 \mathrm{~Hz}), 6.56\left(\mathrm{~m}, 6 \mathrm{H}, \mathrm{H}_{\text {diazine }}\right), 2.25(\mathrm{~d}, 1 \mathrm{H}, \mathrm{J}=5 \mathrm{~Hz}$, $\left.\mathrm{CH}_{3}\right) \cdot{ }^{13} \mathrm{C}-\mathrm{NMR}: \delta(\mathrm{ppm})=160.22(\mathrm{CH}=\mathrm{N}), 55.30$ $\left(\mathrm{OCH}_{3}\right), 22.90\left(\mathrm{CH}_{3}\right), 111.65\left(\mathrm{C}_{1}\right), 167.17\left(\mathrm{C}_{2}, \mathrm{C}_{4}\right)$, $171.90\left(\mathrm{C}_{3}\right), 134.03\left(\mathrm{C}_{5}\right), 129.80\left(\mathrm{C}_{6}, \mathrm{C}_{10}\right), 129.30$ $\left(\mathrm{C}_{7}, \mathrm{C}_{9}\right), 150.90\left(\mathrm{C}_{8}\right), 131.70\left(\mathrm{C}_{11}\right), 130.19\left(\mathrm{C}_{12}, \mathrm{C}_{16}\right)$, $114.40\left(\mathrm{C}_{13}, \mathrm{C}_{15}\right), 158.02\left(\mathrm{C}_{14}\right)(\operatorname{Ar}-\mathrm{C})$. Mass $(\mathrm{m} / \mathrm{z})$ : $396.74(\mathrm{M})+\left[\mathrm{C}_{20} \mathrm{H}_{20} \mathrm{~N}_{4} \mathrm{O}_{3} \mathrm{~S}\right]^{+}$, Base peak = 397.77 $(\mathrm{M}+1)\left[\mathrm{C}_{20} \mathrm{H}_{21} \mathrm{~N}_{4} \mathrm{O}_{3} \mathrm{~S}\right]^{+}, 398.78(\mathrm{M}+2), 400.81(\mathrm{M}+4)$, $363.68\left[\mathrm{C}_{19} \mathrm{H}_{16} \mathrm{~N}_{4} \mathrm{O}_{2} \mathrm{~S}\right]^{+}$, Cal. 396.13; found 396.74. 
4-((4-methoxybenzylidene)amino)-N-(4-methyl pyrimidin-2-yl)benzenesulfonamide[ $\left.\mathrm{L}^{4}\right]$

$\mathrm{C}_{19} \mathrm{H}_{18} \mathrm{~N}_{4} \mathrm{O}_{3} \mathrm{~S}$; Brown colored; yield, 64.92\%; m.p. 204-215 ${ }^{\circ}$; FT-IR $\left(\mathrm{KBr}, \mathrm{Vcm}^{-1}\right): \mathrm{VCH}=\mathrm{N}\left(1590 \mathrm{~cm}^{-}\right.$ $\left.{ }^{1}\right), \mathrm{vNH}\left(3320 \mathrm{~cm}^{-1}\right), \vee \mathrm{CH}_{3}\left(2850 \mathrm{~cm}^{-1}\right), \vee \mathrm{OCH}_{3}(2945$ $\left.\mathrm{cm}^{-1}\right), \vee \mathrm{SO}_{2}\left(1347 \& 1243 \mathrm{~cm}^{-1}\right) .{ }^{1} \mathrm{H}-\mathrm{NMR}: \delta(\mathrm{ppm})=$ $8.53(\mathrm{~S}, 1 \mathrm{H}, \mathrm{CH}=\mathrm{N}), 11.31(\mathrm{~S}, 1 \mathrm{H}, \mathrm{NH}), 3.86(\mathrm{~S}, 3 \mathrm{H}$, $\left.\mathrm{OCH}_{3}\right), 7.08$ (d, 2H, J= 8.7 Hz), 8.31 (dd, $2 \mathrm{H}, \mathrm{J}=1.5$ $\mathrm{Hz}), 7.63(\mathrm{~d}, 2 \mathrm{H}, \mathrm{J}=8.75 \mathrm{~Hz}), 8.00(\mathrm{~d}, 2 \mathrm{H}, \mathrm{J}=8.5 \mathrm{~Hz})$, $7.86(\mathrm{~d}, 1 \mathrm{H}, \mathrm{J}=1.95 \mathrm{~Hz}), 6.95(\mathrm{~m}, 1 \mathrm{H}), 2.32(\mathrm{~d}, 3 \mathrm{H}$, $\left.\mathrm{J}=10 \mathrm{~Hz}, \mathrm{CH}_{3}\right) \cdot{ }^{13} \mathrm{C}-\mathrm{NMR}: \delta(\mathrm{ppm})=160.00(\mathrm{CH}=\mathrm{N})$, $55.57\left(\mathrm{OCH}_{3}\right), 23.22\left(\mathrm{CH}_{3}\right), 111.91\left(\mathrm{C}_{1}\right), 152.84\left(\mathrm{C}_{2}\right)$, $168.90\left(C_{3}\right), 191.18\left(C_{4}\right), 131.68\left(C_{5}\right), 129.36\left(C_{6}, C_{10}\right)$, $124.82\left(C_{7}, C_{9}\right), 156.80\left(C_{8}\right), 129.91\left(C_{11}\right), 130.78\left(C_{12}\right.$, $\left.\mathrm{C}_{16}\right), 114.44\left(\mathrm{C}_{13}, \mathrm{C}_{15}\right), 164.10\left(\mathrm{C}_{14}\right)(\operatorname{Ar}-\mathrm{C})$. Mass $(\mathrm{m} / \mathrm{z})$ : $382.53(\mathrm{M})+\left[\mathrm{C}_{19} \mathrm{H}_{18} \mathrm{~N}_{4} \mathrm{O}_{3} \mathrm{~S}\right]^{+}, 383.82(\mathrm{M}+1), 384.82$ $(\mathrm{M}+2)$, Base peak $=248.64\left[\mathrm{C}_{11} \mathrm{H}_{10} \mathrm{~N}_{3} \mathrm{SO}_{2}\right]^{+}, 367.89[\mathrm{C}$ $\left.{ }_{18} \mathrm{H}_{15} \mathrm{~N}_{4} \mathrm{O}_{3} \mathrm{~S}\right]^{+}$,cal. 382.11; found 382.44.

\section{Synthesis of Sn(II) Complexes}

Tin(II) complexes under investigation were prepared through the reaction of anhydrous tin(II) chloride $(0.001 \mathrm{~mol}, 0.18 \mathrm{~g})$ with sulpha drug based Schiff base ligands $\left(\mathrm{L}^{1}, 0.66 \mathrm{~g}, 0.002 \mathrm{~mol} ; \mathrm{L}^{2}, 0.73\right.$ g, 0.002 mol; L ${ }^{3}, 0.79$ g, 0.002 mol; L4, 0.76 g, 0.002 $\mathrm{mol}$ ) in MeTHF as reaction medium in 1:2 molar ratio in a $100 \mathrm{~mL}$ neat and clean round bottom flask and refluxed on oil bath at $50-65^{\circ} \mathrm{C}$ for about $4-5$ hours. In reaction mixture to adjust $\mathrm{pH}$ 5-6 we have added 2-3 drops of glacial acetic acid. The obtained precipitates were filtered off and washed with cold absolute ethanol 2-3 times and kept in refrigerator for 2-3 days for drying.

Purification of the obtained solid compounds was done by recrystallization using absolute ethanol. Purity of each complex was checked by TLC by using silica gel-G as an adsorbent. Structures of synthesized complexes have been shown in Figure 3.

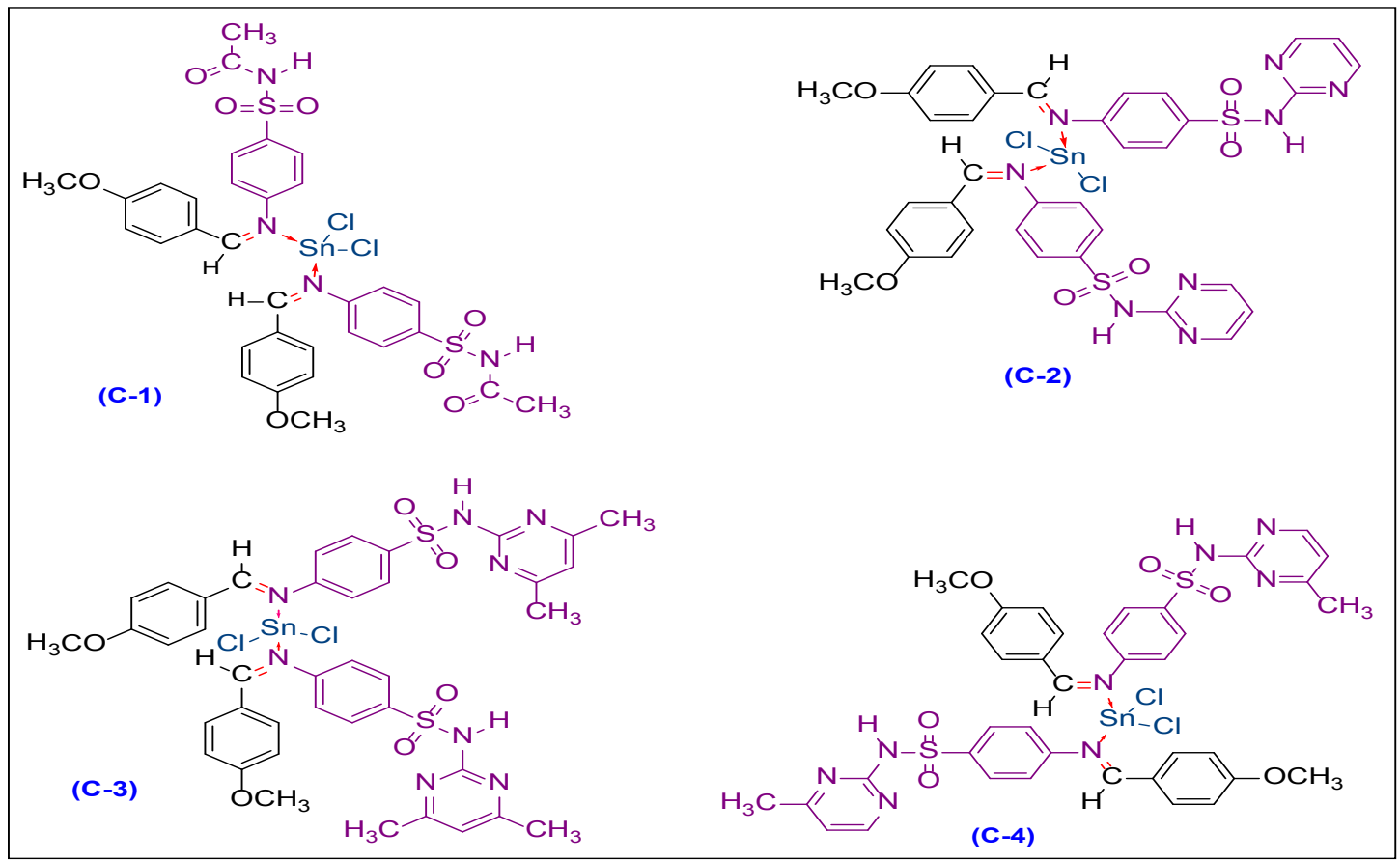

Fig. 3. The proposed structure of tin complexes (C-1 to C-4)

$\left[\mathrm{C}_{33} \mathrm{H}_{34} \mathrm{Cl}_{2} \mathrm{~N}_{4} \mathrm{O}_{8} \mathrm{~S}_{2} \mathrm{Sn}\right](\mathbf{C}-1)$ : Dark brown colour; yield, $73.77 \%$; m.p. $223^{\circ} \mathrm{C}$; FT-IR (KBr, v $\left.\mathrm{cm}^{-1}\right)$ : $\vee \mathrm{CH}=\mathrm{N}\left(1612-1623 \mathrm{~cm}^{-1}\right), \vee \mathrm{C}-\mathrm{O}-\mathrm{C}(1090$ $\left.\mathrm{cm}^{-1}\right), \mathrm{VSO}_{2}\left(1315-1325 \mathrm{~cm}^{-1}\right)$ (Asy.) \& 1145-1150 $\mathrm{cm}^{-1}$ (Sym.). ${ }^{1} \mathrm{H}-\mathrm{NMR}: \delta(\mathrm{ppm})=8.49(\mathrm{~S}, 2 \mathrm{H}$, $\mathrm{CH}=\mathrm{N}), 1.05\left(\mathrm{~S}, 6 \mathrm{H}, \mathrm{CH}_{3}\right), 3.71\left(\mathrm{~S}, 6 \mathrm{H}, \mathrm{OCH}_{3}\right)$, $6.87(\mathrm{~d}, 4 \mathrm{H}, \mathrm{J}=3.5 \mathrm{~Hz}), 7.48(\mathrm{dd}, 4 \mathrm{H}, \mathrm{J}=5 \mathrm{~Hz})$, $7.66(\mathrm{~d}, 4 \mathrm{H}, \mathrm{J}=8.5 \mathrm{~Hz}), 6.58(\mathrm{~d}, 4 \mathrm{H}, \mathrm{J}=8.5 \mathrm{~Hz})$.
${ }^{13} \mathrm{C}-\mathrm{NMR}: \delta(\mathrm{ppm})=169.3(\mathrm{CH}=\mathrm{N}), 206.42(\mathrm{C}=\mathrm{O})$, $55.92\left(\mathrm{COCH}_{3}\right), 18.43\left(\mathrm{CH}_{3}\right), 137.7(\mathrm{C} 1), 116.3$ $\left(\mathrm{C}_{2}, \mathrm{C}_{6}\right), 127.30\left(\mathrm{C}_{3}, \mathrm{C}_{5}\right), 130.09\left(\mathrm{C}_{4}\right), 122.12\left(\mathrm{C}_{7}\right)$, $123.86\left(\mathrm{C}_{8}, \mathrm{C}_{12}\right), 114.35\left(\mathrm{C}_{9}, \mathrm{C}_{11}\right), 168.7\left(\mathrm{C}_{10}\right)(\mathrm{Ar}-$ C). Mass (m/z): 868.42(M)+, $870.34(\mathrm{M}+2), 872.80$ $(\mathrm{M}+4), 851.85\left[\mathrm{C}_{33} \mathrm{H}_{33} \mathrm{Cl}_{2} \mathrm{~N}_{4} \mathrm{O}_{7} \mathrm{~S} 2 \mathrm{Sn}\right]^{+}, 904.22$ $\left[\mathrm{C}_{33} \mathrm{H}_{38} \mathrm{Cl}_{2} \mathrm{~N}_{4} \mathrm{O}_{10} \mathrm{~S}_{2} \mathrm{Sn}\right]^{+}, 905.75\left[\mathrm{C}_{33} \mathrm{H}_{39} \mathrm{Cl}_{2} \mathrm{~N}_{4} \mathrm{O}_{10} \mathrm{~S}_{2} \mathrm{Sn}\right]$, cal. 868.04; found 868.42. 
$\left[\mathrm{C}_{36} \mathrm{H}_{32} \mathrm{Cl}_{2} \mathrm{~N}_{8} \mathrm{O}_{6} \mathrm{~S}_{2} \mathrm{Sn}\right](\mathrm{C}-2)$ : Red colour; yield, $52 \%$; m.p. $252^{\circ} \mathrm{C}$; FT-IR $\left(\mathrm{KBr}, \mathrm{vcm}^{-1}\right): \mathrm{v}(\mathrm{CH}=\mathrm{N})$ $\left(1605 \mathrm{~cm}^{-1}\right), \mathrm{v} \mathrm{NH}\left(1070 \mathrm{~cm}^{-1}\right) .{ }^{1} \mathrm{H}-\mathrm{NMR}: \delta(\mathrm{ppm})=$ $8.00(\mathrm{~S}, 2 \mathrm{H}, \mathrm{CH}=\mathrm{N}), 11.66(\mathrm{~S}, 2 \mathrm{H}, \mathrm{NH}), 3.81(\mathrm{~S}$, $\left.6 \mathrm{H}, \mathrm{OCH}_{3}\right), 6.90-8.00(\mathrm{~m}, 16 \mathrm{H}$, Aryl), $8.48(\mathrm{~d}, 4 \mathrm{H}$, $\left.\mathrm{H}_{\text {diazine }}\right), 7.02\left(\mathrm{t}, 2 \mathrm{H}, \mathrm{H}_{\text {diazine }}\right) \cdot{ }^{13} \mathrm{C}-\mathrm{NMR}: \delta(\mathrm{ppm})=174.2$ $(\mathrm{CH}=\mathrm{N}), 55.80\left(\mathrm{OCH}_{3}\right), 169.3\left(\mathrm{C}_{1}\right), 157.9\left(\mathrm{C}_{2}, \mathrm{C}_{4}\right)$, $115.3\left(\mathrm{C}_{3}\right), 139.7\left(\mathrm{C}_{5}\right), 127.3\left(\mathrm{C}_{6}, \mathrm{C}_{10}\right), 125.7\left(\mathrm{C}_{7}, \mathrm{C}_{9}\right)$, $147.1\left(C_{8}\right), 122.7\left(C_{11}\right), 124.0\left(C_{12}, C_{16}\right), 114.3\left(C_{13}\right.$ $\left.\mathrm{C}_{15}\right), 160.6\left(\mathrm{C}_{14}\right)(\operatorname{Ar}-\mathrm{C})$. Mass (m/z): cal. 926.05; found 926.46 .

$\left[\mathrm{C}_{42} \mathrm{H}_{44} \mathrm{Cl}_{2} \mathrm{~N}_{8} \mathrm{O}_{6} \mathrm{~S}_{2} \mathrm{Sn}\right](\mathrm{C}-3)$ : Orange colour; yield, $79.80 \%$; m.p. $309^{\circ} \mathrm{C}$; FT-IR $\left(\mathrm{KBr}, \mathrm{V} \mathrm{cm}^{-1}\right): \mathrm{V}$ $\mathrm{CH}=\mathrm{N}\left(1598 \mathrm{~cm}^{-1}\right), \mathrm{v} \mathrm{NH}\left(1066 \mathrm{~cm}^{-1}\right) .{ }^{1} \mathrm{H}-\mathrm{NMR}: \delta$ $(\mathrm{ppm})=8.50(\mathrm{~S}, 2 \mathrm{H}, \mathrm{CH}=\mathrm{N}), 10.95(\mathrm{~S}, 2 \mathrm{H}, \mathrm{NH}), 3.81$ $\left(\mathrm{S}, 6 \mathrm{H}, \mathrm{OCH}_{3}\right), 2.49\left(\mathrm{~d}, 12 \mathrm{H}, \mathrm{J}=1.5 \mathrm{~Hz}, \mathrm{CH}_{\text {3diazine }}\right)$, $7.66\left(\mathrm{~m}, 2 \mathrm{H}, \mathrm{H}_{\text {diazine }}\right), 7.01(\mathrm{~d}, 4 \mathrm{H}, \mathrm{J}=8.7 \mathrm{~Hz}), 6.56$ (d, $4 \mathrm{H}, \mathrm{J}=8.7 \mathrm{~Hz}), 6.55-6.93\left(\mathrm{~m}, 8 \mathrm{H}\right.$, Aryl). ${ }^{13} \mathrm{C}-\mathrm{NMR}$ : $\delta(\mathrm{ppm})=81.26(\mathrm{CH}=\mathrm{N}), 23.02\left(\mathrm{CH}_{3}\right), 160.6\left(\mathrm{C}_{1}\right)$, $114.3\left(\mathrm{C}_{2}, \mathrm{C}_{6}\right), 124.0\left(\mathrm{C}_{3}, \mathrm{C}_{5}\right), 122.7\left(\mathrm{C}_{4}\right), 143.10$ $\left(C_{7}\right), 125.7\left(C_{8}, C_{12}\right), 127.3\left(C_{9}, C_{11}\right), 168.5\left(C_{13}\right)$, $166.6\left(\mathrm{C}_{14}, \mathrm{C}_{16}\right), 111.88\left(\mathrm{C}_{15}\right), 130.19\left(\mathrm{C}_{10}\right)(\mathrm{Ar}-\mathrm{C})$. Mass (m/z): $1010.96(\mathrm{M})^{+}\left[\mathrm{C}_{42} \mathrm{H}_{44} \mathrm{Cl}_{2} \mathrm{~N}_{8} \mathrm{O}_{6} \mathrm{~S}_{2} \mathrm{Sn}\right]^{+}$, $1012.25[\mathrm{M}+2]\left[\mathrm{C}_{42} \mathrm{H}_{46} \mathrm{Cl}_{2} \mathrm{~N}_{8} \mathrm{O}_{6} \mathrm{~S}_{2} \mathrm{Sn}\right]^{+}, 1014.58[\mathrm{M}+4]$ $\left[\mathrm{C}_{42} \mathrm{H}_{48} \mathrm{Cl}_{2} \mathrm{~N}_{8} \mathrm{O}_{6} \mathrm{~S}_{2} \mathrm{Sn}\right]^{+}, 995.85\left[\mathrm{C}_{41} \mathrm{H}_{41} \mathrm{Cl}_{2} \mathrm{~N}_{8} \mathrm{O}_{6} \mathrm{~S}_{2} \mathrm{Sn}\right]^{+}$, $996.83\left[\mathrm{C}_{42} \mathrm{H}_{44} \mathrm{Cl}_{2} \mathrm{~N}_{7} \mathrm{O}_{6} \mathrm{~S}_{2} \mathrm{Sn}\right]^{+}, 1046.73\left[\mathrm{C}_{42} \mathrm{H}_{48} \mathrm{Cl}_{2} \mathrm{~N}\right.$ $\left.{ }_{8} \mathrm{O}_{8} \mathrm{~S}_{2} \mathrm{Sn}\right]^{+}, 1048.88\left[\mathrm{C}_{42} \mathrm{H}_{50} \mathrm{Cl}_{2} \mathrm{~N}_{8} \mathrm{O}_{8} \mathrm{~S}_{2} \mathrm{Sn}\right]^{+}, 1050.94$ $\left[\mathrm{C}_{42} \mathrm{H}_{52} \mathrm{Cl}_{2} \mathrm{~N}_{8} \mathrm{O}_{8} \mathrm{~S}_{2} \mathrm{Sn}\right]^{+}, 1031.30\left[\mathrm{C}_{41} \mathrm{H}_{41} \mathrm{Cl}_{2} \mathrm{~N}_{8} \mathrm{O}_{8} \mathrm{~S}_{2} \mathrm{Sn}\right]^{+}$, $1016.85\left[\mathrm{C}_{41} \mathrm{H}_{46} \mathrm{Cl}_{2} \mathrm{~N}_{8} \mathrm{O}_{7} \mathrm{~S}_{2} \mathrm{Sn}\right]^{+}$, Cal. 1010.14; found 1010.96 .

$\left[\mathrm{C}_{38} \mathrm{H}_{36} \mathrm{Cl}_{2} \mathrm{~N}_{8} \mathrm{O}_{6} \mathrm{~S}_{2} \mathrm{Sn}\right](\mathbf{C}-4)$ : Mud colour; yield, $65.50 \%$; m.p. $310^{\circ} \mathrm{C}$; FT-IR $\left(\mathrm{KBr}, \mathrm{v} \mathrm{cm}^{-1}\right)$ : $\mathrm{V}$ $\mathrm{CH}=\mathrm{N}\left(1585 \mathrm{~cm}^{-1}\right), \vee \mathrm{NH}\left(1066 \mathrm{~cm}^{-1}\right) .{ }^{1} \mathrm{H}-\mathrm{NMR}$ : $\delta(\mathrm{ppm})=8.00(\mathrm{~S}, 2 \mathrm{H}, \mathrm{CH}=\mathrm{N}), 11.68(\mathrm{~S}, 2 \mathrm{H}, \mathrm{NH})$, $3.81\left(\mathrm{~S}, 6 \mathrm{H}, \mathrm{OCH}_{3}\right), 2.50\left(\mathrm{t}, 6 \mathrm{H}, \mathrm{CH}_{3}\right), 6.93-9.20(\mathrm{~m}$, $16 \mathrm{H}$, aryl), $8.11\left(\mathrm{q}, 4 \mathrm{H}, \mathrm{H}_{\text {diazine }}\right) \cdot{ }^{13} \mathrm{C}-\mathrm{NMR}: \delta(\mathrm{ppm})=$ $168.9(\mathrm{CH}=\mathrm{N}), 55.8\left(\mathrm{OCH}_{3}\right), 23.90\left(\mathrm{CH}_{3}\right), 170.1\left(\mathrm{C}_{1}\right)$, $110.1\left(\mathrm{C}_{2}, \mathrm{C}_{4}\right), 168.9\left(\mathrm{C}_{3}\right), 147.1\left(\mathrm{C}_{5}\right), 124.0\left(\mathrm{C}_{6}, \mathrm{C}_{10}\right)$, $127.3\left(C_{7}, C_{9}\right), 139.7\left(C_{8}\right), 125.7\left(C_{11}\right), 127.3\left(C_{12}\right.$, $\left.\mathrm{C}_{16}\right), 114.3\left(\mathrm{C}_{13}, \mathrm{C}_{15}\right), 160.6\left(\mathrm{C}_{14}\right)(\operatorname{Ar}-\mathrm{C})$. Mass $(\mathrm{m} / \mathrm{z})$ : Cal. 954.08; found 954.52.

\section{Antibacterial and Antifungal Activity}

Antibacterial activity of ligands $\left(L^{1}-L^{4}\right)$ as well as their $\mathrm{Sn}$ (II) complexes (C-1 to $\mathrm{C}-4$ ) have studied by Broth dilution method ${ }^{35,36}$ and MIC values were calculated. Following bacterial strains viz. $S$. aureus, S. pyogenus (Gram-positive) and E. coli, $P$. aeruginosa (Gram-negative) were used. These strains were procured from institute of microbial technology, Chandigarh, India. Dimethyl sulfoxide solvent was used for serial dilution to receive the appropriate concentration of drugs.

In this method, stock solution of concentration $2000 \mu \mathrm{g} / \mathrm{mL}$ was prepared. In primary screening, the stock solution was diluted to give $1000,500,250 \mu \mathrm{g} / \mathrm{mL}$ concentration and screened for their activity and active drug then screened in secondary screening with $200,100,50,25,12.5$, $6.2 \mu \mathrm{g} / \mathrm{mL}$ concentration. After serial dilution the control tube with lowest concentration was taken. The control tubes without antibiotic were immediately subcultured by uniform spreading over a quarter of plate of medium with the help of loop for growth of the test organism and control tubes incubated overnight at $37^{\circ} \mathrm{C}$ temperature. After incubation, turbidity was measured and microbial growth in the control tubes was compared. The control tubes before incubation represented the original inoculums. The effect of concentration of drug was checked after incubation and the control tube with lowest concentration of drug (lack of turbidity), was shown better inhibition in growth of bacteria. Inoculums size was adjusted to $10^{8} \mathrm{Cfu} / \mathrm{mL}$ for bacterial test strain by comparing the turbidity in the tubes.

The antifungal activities of the synthesized compounds were tested against fungal strains C. albicans, A. niger, A. clavatus and MIC values were determined. SDB (Sabourand dextrose broth) medium was used for the fungal nutrition, which contains $10 \mathrm{~g}$ peptone (from casein), $40 \mathrm{~g}$ dextrose (glucose), $1 \mathrm{~L}$ of distilled water, $\mathrm{pH}$ adjusted to 5.6 at $25^{\circ} \mathrm{C}^{37}$. Essential amount of the compounds was added into this medium in DMSO. Serial dilutions were prepared for primary and secondary screening as discussed in previous section. The control tubes were kept for incubation for $72 \mathrm{~h}$ at $22^{\circ} \mathrm{C}$ and MIC were recorded. Standard drug Greseofulvin was used.

\section{Antimalarial Activity}

In vitro antimalarial activities of all ligands and their Sn(II) complexes were evaluated against $P$. falciparum strain. In antimalarial activity we have used 96 well microtitre plates. The cultures of $P$. falciparum strain were maintained in the medium RPMI 1640 supplemented with 1\% D-glucose, $25 \mathrm{Mm}$ HEPS, $\mathrm{NaHCO}_{3} 0.23 \%$ and $10 \%$ heat inactivated human serum. In this method, prepared 
a stock solution of $(5 \mu \mathrm{g} / \mathrm{mL})$ of all test samples using DMSO.

Serial dilutions were prepared with culture medium in same solvent. $20 \mu \mathrm{L}$ of the samples were diluted at five fold to give $0.4 \mu \mathrm{g} / \mathrm{mL}$ to 100 $\mu \mathrm{g} / \mathrm{mL}$ concentration which were kept in the culture plates for incubation at $37^{\circ} \mathrm{C}$ for $36-40 \mathrm{~h}$ into a candle jar. Thin blood smears were prepared after incubation from each well then stained it with JSB stain. To record maturation of ring stage parasites into trophozoites and schizonts, slides were microscopically examined ${ }^{38-43}$ and percent inhibition in maturation, average rings of trophozoites and schizontes were recorded per 100 parasities from the duplicate wells. Results were expressed as $I C_{50}$ value as depicted in Table 1.

\section{RESULTS AND DISCUSSION}

\section{Preparation \& Characterization of Sn(II) complexes}

[Sn $(\mathrm{L})_{2} \mathrm{Cl}_{2}$ ] type of complexes were prepared through the reaction of anhydrous tin dichloride with bi-dentate sulpha drug ligands in 1:2 molar ratio using three different solvents ethanol, MeTHF and THF were used and MeTHF solvent was found with best results. The reaction found to be quite facile and could be completed in 4-5 h of refluxing on oil bath. All newly synthesized complexes were obtained in the form of colored solid and the color of the complexes are associated with the presence of chromophoric azomethine group $(>\mathrm{C}=\mathrm{N}-)$.
FT-IR gives the information about functional group attached to metal atom ${ }^{44}$. In FT-IR spectrum of all the complexes azomethine peak shifted towards lower frequency in Sn(II) complexes that represent bonding of azomethine nitrogen with metal ion. In the IR spectrum of ligands, stretching vibrations of $>\mathrm{C}=\mathrm{O}$ group appeared at $1700-1720 \mathrm{~cm}^{-1}$ and $\mathrm{SO}_{2}$ group appeared at $1315-1350 \mathrm{~cm}^{-1}$ (asymmetric) \& $1150-1247 \mathrm{~cm}^{-1}$ (symmetric) are not changed on complexation which indicates both groups $(>\mathrm{C}=\mathrm{O}$ $\& \mathrm{SO}_{2}$ ) are not involved in coordination with metal. Two new bands appeared around at 519-555 $\mathrm{cm}^{-1}$ and $410 \mathrm{~cm}^{-1}$ due to $v \mathrm{M}-\mathrm{N}$ and $\mathrm{v} \mathrm{M}-\mathrm{Cl}$ bonds confirm metal-ligand bonding, respectively.

\section{Thermogravimetric Analysis (TGA)}

The thermal decomposition analysis of synthesized complex C-3 was carried out under an inert nitrogen atmosphere (Fig. 4). A weight loss of $8 \%$ was observed between $100-230^{\circ} \mathrm{C}$ due to loss of two chlorine atoms. Another thermal decomposition occurs between $230-882^{\circ} \mathrm{C}$ with weight loss of $52 \%$ due to loss of remaining organic part. Total $60 \%$ weight loss was observed between $100-882^{\circ} \mathrm{C}$. Heating rate was suitably controlled at $30^{\circ} \mathrm{C}$ minute ${ }^{-1}$.

Thus TGA curve indicates presence of two chlorine atoms in the tin complex and tetrahedral geometry has been suggested having 1:2 stiochiometric ratio of $\mathrm{Sn}(\mathrm{II})$ and ligand, respectively.

\section{Differential Thermal Analysis (DTA)}

In DTA curve of C-3 complex two downward peaks around $250-290^{\circ} \mathrm{C}$ and $450-470^{\circ} \mathrm{C}$ were observed which indicate reaction is endothermic in nature.
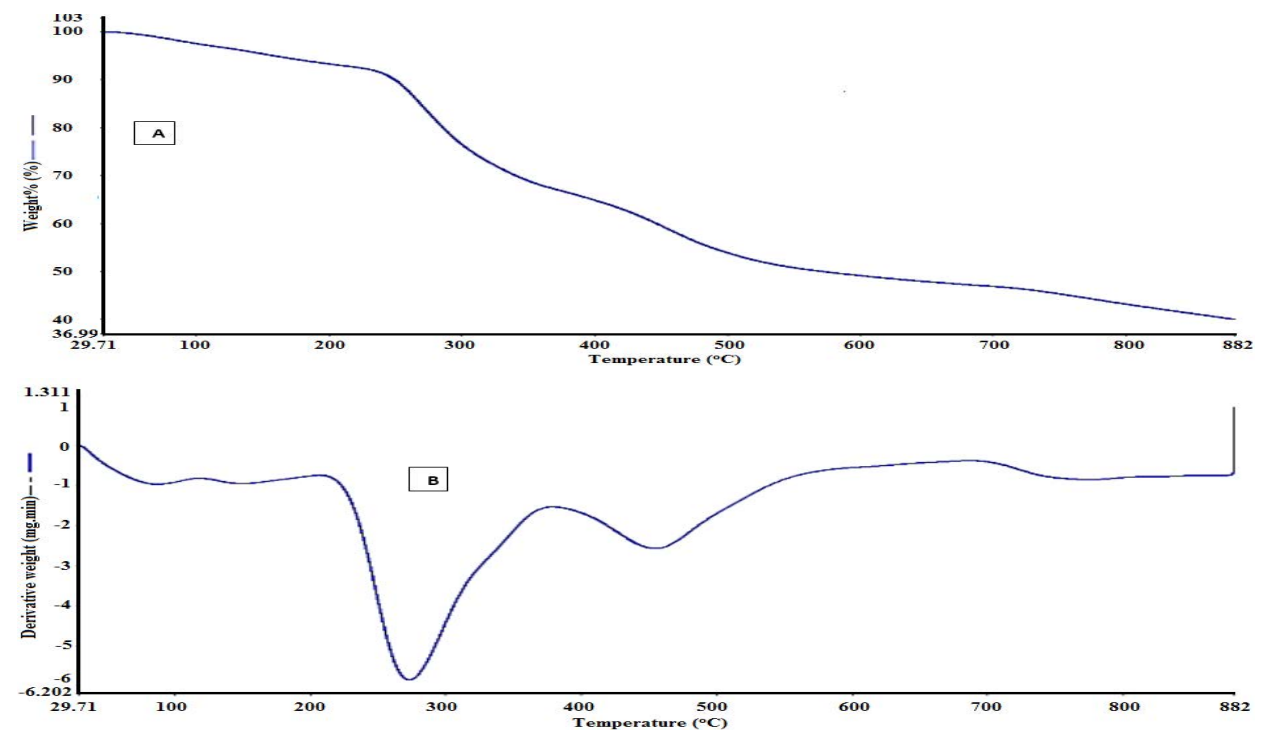

Fig. 4.(a) TGA curve (b) DTA curve for C-3 complex 
${ }^{1} \mathrm{H}-\mathrm{NMR}$ of azomethine nitrogen $(-\mathrm{CH}=\mathrm{N}-)$ showed upfield shifting from $\delta 8.52-8.55$ to 8.00 $8.50 \mathrm{ppm}$ in their tin complexes. This upfield shifting in complex indicate discharging of lone pair of nitrogen atom towards the metal ion. Methoxy proton appeared at $\delta 3.86-3.65 \mathrm{ppm}$ as singlet, this indicates methoxy group is not involved in coordination with metal ion.

All aromatic protons shifted towards downfield region on complexation with the Sn metal ion. In C-1 and C-3 complexes ortho protons of aryl shifted towards downfield region at $\delta 7.38$ to 7.66 ppm and $\delta 6.78$ to $7.01 \mathrm{ppm}$, respectively due to complexation with metal.

The proposed structures (Fig. 3.) of tin(II) complexes (C-1 to C-4) is in good agreement with the spectral data presented and reported literature ${ }^{45-46}$. In ${ }^{13}$ C-NMR spectra of C-3 complex, a downfield shifting was observed in the azomethine carbons from $\delta 158.14$ $162.39 \mathrm{ppm}$ (free ligands) to $\delta 169.3-174.2 \mathrm{ppm}$ (tin complexes) may be due to shifting of lone pair of nitrogen towards metal ion. Similarly all phenyl ring carbons shifted towards downfield region by $0.1-0.40 \mathrm{ppm}$.

Mass fragmentation pattern of all Schiff base ligands $\left(L^{1}-L^{4}\right)$ and their complexes $(C-1$ to $C-4)$ were recorded. LC-MS of Schiff base $L^{3}$ and its tin complex C-3 were explained here. The molecular ion peaks at $\mathrm{m} / \mathrm{e} 396.74$ and 1010.96 attributed to the ligand \& its complex, respectively. Mass spectra of the complex C-3 showed peaks at $\mathrm{m} / \mathrm{e} 1010.96,1012.25$, $1014.58,1031.30$ and 1016.85 corresponding to $\left(\mathrm{M}^{+}\right),(\mathrm{M}+2),(\mathrm{M}+4),\left[\mathrm{C}_{41} \mathrm{H}_{41} \mathrm{Cl}_{2} \mathrm{~N}_{8} \mathrm{O}_{6} \mathrm{~S} \mathrm{Sn}^{+}\right.$and $\left[\mathrm{C}_{41} \mathrm{H}_{42} \mathrm{Cl}_{2} \mathrm{~N}_{8} \mathrm{O}_{5} \mathrm{~S}_{2} \mathrm{Sn}\right]^{+}$, respectively and confirms that complex contains two chlorine atoms.

\section{Antibacterial Activity}

Results of antibacterial activity indicate that the C-3 complex was found most active (MIC, $50 \mu \mathrm{g} /$ $\mathrm{mL}$ ) against the pathogen $E$. coli and C-1 complex was found with good activity (MIC, $55.6 \mu \mathrm{g} / \mathrm{mL}$ ) against $E$. coli. Further $\mathrm{C}-2$ was found more active and C-3, C-4 was found with good activity against Gram-negative P. aeruginosa. C-1, C-2 and C-3 complexes were found with good activity against $S$. aureus bacteria. Complex $\mathrm{C}-1$ was found with better activity against $S$. pyogenus bacteria. $\mathrm{L}^{3}$ was found with better activity (MIC, $52 \mu \mathrm{g} / \mathrm{mL}$ ) against the pathogen $E$. coli. Thus these results indicate that complexes exhibit better activity than the ligands.

\section{Antifungal Activity}

The antifungal activity of four Schiff base ligands $\left(\mathrm{L}^{1}-\mathrm{L}^{4}\right)$ and their complexes (C-1 to $\left.\mathrm{C}-4\right)$ were tested against fungal strains $C$. albicans, $A$. niger and $A$. clavatus. Complex $\mathrm{C}-3$ exhibit better activity (MIC, $250 \mu \mathrm{g} / \mathrm{mL}$ ) and complex C-1 was found with good activity (MIC, $500 \mu \mathrm{g} / \mathrm{mL}$ ) against $C$. albicans. C-3 complex was found with better activity (MIC, $100 \mu \mathrm{g} / \mathrm{mL}$ ) and C-1, C-2, C-4 complexes showed good activity against $A$. Niger. C-4 complex was found with good activity (MIC, $250 \mu \mathrm{g} / \mathrm{mL}$ ) against A. clavatus bacteria. Greseofulvin drug was used against the bacteria. $\mathrm{L}^{3}$ and $\mathrm{L}^{4}$ showed with good activity against $A$. niger and $C$. albicans (MIC, 250 $\mu \mathrm{g} / \mathrm{mL}$ ). These results indicate that the complexes show good activity than the ligands.

\section{Antimalarial Activities}

In vitro antimalarial activities of ligands $\left(L^{1}-L^{4}\right)$ and their complexes (C-1 to C-4) were evaluated against parasite $P$. falciparum strain. Results of antimalarial activity were expressed as $I_{50}$ value (Table 1). The smaller $\mathrm{IC}_{50}$ value indicates higher the antimalarial activity. Chloroquine was used as control drug in this study. Complex $\mathrm{C}-3$ was found more active against chloroquine $\left(\mathrm{IC}_{50}, 0.04 \mu \mathrm{g} / \mathrm{mL}\right)$ and complex C-1 was found with good activity $\left(\mathrm{IC}_{50}, 0.07 \mu \mathrm{g} / \mathrm{mL}\right)$ than other complexes. $\mathrm{L}^{3}$ showed with good activity against chloroquine $\left(\mathrm{IC}_{50}, 0.10 \mu \mathrm{g} / \mathrm{mL}\right)$. These results indicate that complexes are more significant than the ligands.

Table 1: Result of antibacterial, antifungal and antimalarial activities of Schiff base ligands $\left(L^{1}-L^{4}\right)$ and complexes (C-1 to C-4)

\begin{tabular}{|c|c|c|c|c|c|c|c|c|c|}
\hline \multirow{2}{*}{\multicolumn{2}{|c|}{ S. No. Compound }} & \multicolumn{3}{|c|}{ Antibacterial activity (MIC, $\mu \mathrm{g} / \mathrm{mL}$ ) } & \multicolumn{5}{|c|}{ Antifungal activity (MIC, $\mu \mathrm{g} / \mathrm{mL}$ ) } \\
\hline & & $\begin{array}{c}\text { E. coli } \\
\text { (MTCC 443) }\end{array}$ & $\begin{array}{l}\text { P. aeruginosa } \\
\text { (MTCC 441) }\end{array}$ & $\begin{array}{l}\text { S. aureus } \\
\text { (MTCC 96) }\end{array}$ & $\begin{array}{l}\text { S. pyogenus } \\
\text { (MTCC 442) }\end{array}$ & $\begin{array}{l}\text { C. albicans } \\
\text { (MTCC 227) }\end{array}$ & $\begin{array}{c}\text { A. niger } \\
\text { (MTCC 282) }\end{array}$ & $\begin{array}{c}\text { A. clavatus } \\
\text { (MTCC 1323) }\end{array}$ & P. falciparum \\
\hline 1 & $L^{1}$ & 62.5 & 100 & 100 & 100 & 1000 & 500 & 1000 & $0.14 \mu \mathrm{g} / \mathrm{mL}$ \\
\hline 2 & $\mathrm{~L}^{2}$ & 100 & 125 & 125 & 250 & 500 & 500 & 1000 & $1.36 \mu \mathrm{g} / \mathrm{mL}$ \\
\hline 3 & $\mathrm{~L}^{3}$ & 52 & 125 & 61.3 & 100 & 500 & 250 & 500 & $0.10 \mu \mathrm{g} / \mathrm{mL}$ \\
\hline 4 & $\mathrm{~L}^{4}$ & 125 & 250 & 100 & 125 & 250 & 250 & 500 & $0.19 \mu \mathrm{g} / \mathrm{mL}$ \\
\hline 5 & C-1 & 55.6 & 100 & 65.5 & 85.2 & 500 & 250 & 1000 & $0.07 \mu \mathrm{g} / \mathrm{mL}$ \\
\hline 6 & C-2 & 100 & 62.5 & 100 & 125 & 500 & 250 & 500 & $0.90 \mu \mathrm{g} / \mathrm{mL}$ \\
\hline 7 & $C-3$ & 50 & 100 & 51.5 & 100 & 250 & 100 & 500 & $0.04 \mu \mathrm{g} / \mathrm{mL}$ \\
\hline \multirow[t]{2}{*}{8} & C-4 & 250 & 125 & 100 & 250 & 250 & 200 & 250 & $0.40 \mu \mathrm{g} / \mathrm{mL}$ \\
\hline & $\begin{array}{l}\text { Standard } \\
\text { drugs }\end{array}$ & 25 & 25 & 50 & 50 & 500 & 100 & 100 & $0.020 \mu \mathrm{g} / \mathrm{mL}$ \\
\hline
\end{tabular}


Structure Activity Relationship (SAR) studies

To look at the structure activity relationship (SAR) of sulpha drug Schiff base ligands, the variations were determined on one end of sulphonamide moiety with six membered two heteroatoms with alkyl groups. On the other hand azomethine linkage was responsible for biological activity. Among the four complexes (C-1 to C-4), complex C-3 was found to be best antibacterial, antifungal and antimalarial agent among the tested compounds due to sulphadiazine moiety and two methyl substituents.

Moreover, comparative study of biological activities of all the tested Schiff bases and their complexes revealed that $\mathrm{Sn}$ (II) complexes are more active than respective ligands.

\section{CONCLUSION}

In our present studies, sulpha drugs based Schiffbases and their Sn(II) complexes (C-1 to C-4) were prepared in MeTHF by conventional method and characterized by various techniques. Thus Schiff bases can bind with metal ion through $\mathrm{N}$-atom and tetrahedral geometry is proposed. All the Schiff base ligands and their complexes were screened for antibacterial, antifungal and antimalarial activities. Complex having sulphadiazene moiety has presented good antibacterial, antifungal and antimalarial activity. It was concluded that $\mathrm{Sn}$ (II) complexes are more active as compare to respective ligands.

\section{ACKNOWLEDGEMENT}

Kiran Meena is highly thankful to SAIF, Punjab University, Chandigarh for NMR \& MASS analysis and for IR spectra, Department of Chemistry, M. L. S. U. Udaipur (Raj.), India. For biological studies authors are also grateful to Microcare Laboratory, Surat (Gujrat), India.

\section{Conflicts of Interest}

The authors declare no conflict of interest.

\section{REFERANCES}

1. Abbas, S.A.; Munir, M.; Fatima, A.;Naheed, S.; llyas, Z.; Electronic J. of Life Science., 2010, 1, 37-40.

2. El-Mossalamy, E.H.; Al-Thabati, S.A.; Al-Nowalser, F.M.; Al-Sulami, Q.A.; Communications Faculty of Sciences University of Ankara Series B., 2005, 51, 21-30.

3. Li, Y.; Yang, Z.S.; Zhang, H.; Cao, B.J.; Wang, F.D.; Bioorg.\&Med. Chem., 2003, 11, 4363-4368.

4. Villar, R.; Encio, I.; Migliaccio, M.; Gil, M.G.; Martinez-Merino,V.; Bioorg.\& Med. Chem., 2004, 12, 963- 968.

5. Panneerselvam, P.; Nair, R.R.; Vijayalakshmi, G.; Subramanian, E.H.; Sridhar, S.K.; Eur. J. Med. Chem., 2005, 4O(2), 225-229.

6. Bhat, M.A.; Imran, M.; Khan, S.A.; Siddiqui,N.J.; Pharma. Sci., 2005, 67, 151-159.

7. Wang, L.; Feng, Y.; Xue, J.; Li, Y. J.; Serbian Chem. Soc., 2008, 73, 1-6.

8. Wadher, S.J.; Puranik, M.P.; Karande, N.A.; Yeole, P.G.; Int. J. Pharm. Tech. Res., 2009, 1, 22-33.

9. Matela, G.; Anti-cancer agents in medicinal chemistry., 2020, 20, 1908-1917.

10. Zoubi, W.A.; Int. J. of. Org. Chem., 2013, 3, 73-95.

11. Prakash, A.; Adhikari, D. J.; Pharm. Tech.
Res., 2011, 3-4, 1891-1896.

12. Mostafa, A.A.; Al-Askar, A.A.; Almaary, K.S.; Dawoud, T.M.; Sholkamy, E.N.; Bakri, M.M.; Saudi J. of Biological Sciences., 2018, 25, 361-366.

13. Balouiri, M.; Sadiki, M.; Ibnsouda, S.K; J. of pharma. Analysis., 2016, 6, 71-79.

14. Silva, C.M.; Silva, D.L; Modolo, L.V.; Alves, R.B.; Reseude, M.A.; Martins, C.V.B.; Fatima, A.D.; J. of Adv. Res., 2011, 2, 1-8.

15. Chukwujekwu, J.C.; Staden, J.V.; Smith, P.; South African. J. of Botany., 2005, 71, 316-325.

16. Rudrapal, M.; Chetia, D.; Prakash, A.; Med. Chem. Res., 2013, 22, 3703-3711.

17. Chauhan, L.K.; Nimodia, K.; Ranawat, P.S.; Goswami, A.K.; Baroliya, P.K.; Orient. J. Chem., 2020, 36(5), 855-862.

18. Regar, M.; Baroliya, P.K.; Patidar, A.; Dashora, R.; Mehta, A.; Chauhan, R.S.; Goswami, A.K.; Pharmaceutical Chemistry journal., 2016, 50, 5.

19. Dayma, V.; Chopra, J.; Sharma, P.; Dwivedi, A.; Tripathi, I.P.; Bhargava, A.; Murugesan, V.; Goswami, A.K.; Baroliya, P.K.; Heliyon., 2020, 6, 4787.

20. Patil, U.; Khan, A.; Nagarsekar, A.; Mandewale, M.; Yamgar, R.; Orient. J. Chem., 2018, 34(6), 2796-2805. 
21. (a) Supuran, C.T.; Casini, A.; Scozzafava, A.; Med. Res. Rev., 2003, 5, 535. (b) Scozzafava, A.; Owa,T.; Mastrolorenzo, A.; Supuran,C.T.; Curr. Med. Chem., 2003, 10, 925.

22. Kleemann, A.; Engel, J.; Kutscher, B.; Reichert, D.; Eds. Pharmaceutical Substances, Syntheses, Patents, Applications., (Thieme, Stuttgart., 1999).

23. Domagk, G. Chemotherapy of bacterial infections. Dtsch. Med. Wochensch., 1935, 61, 250-253.

24. Domagk G. A new class of disinfectants. Dtsch. Med. Wochensch., 1935, 61, 829-832.

25. Domagk G., Chemotherapy of bacterial infections. Angew. Chem., 1935, 48, 657-667.

26. Mahmood-ul,H.; J. Enzyme Inhibition \& Med. Chem., 2004, 19, 263-267.

27. Singh,V.; Kaushik, N.K.; Singh,R.; Asian J. Research Chem., 2011, 4(3), 339-347.

28. Brodowska, K.; Charucinska, E.L.; Chemik., 2014, 68, 129-134.

29. Davies,A.G.; Smith,P.J.; Wilkinson,G.; Stone,F.G.A.; Abel, E.W.; Comprehensive Org. Chem., 1982, 2, 519.

30. Priyanka; Kumar, M.; Sharma, H.K.; Soni, S.; Orient. J. Chem., 2020, 36(5), 871-878.

31. Shekhawat, V.S.; Varshney, S.; Varshney, A.K.; J. Indian Chem. Soc., 2017, 94, 21-28.

32. Armarego, W. L. F.; Perin, D. D. $4^{\text {th }}$ ed., Butterworth Heinemann., 1997.

33. Barnabas, M. J.;Parambadath, S.; Nagappan, S.; Ha, C. S.; Heliyon., 2019, 5, 2405-8440.

34. Hassan, M.; Nasr, S.M.; Razak, S. E. A. E.; Aziz, M. S. A.; Gamasy, S.M.E.; Arabian J. of. Chem., 2020, 13, 7324-7337.

35. Clinical and Laboratory Standards Institute Methods for antimicrobial susceptibility testing of aerobic bacteria approved standard M07- A8, 9th edn. National Committee for Clinical Laboratory Standards, Wayne., 2008.

36. Muller, J. H.; Hinton, J.; Proc. Soc. Exptl. Biol., 1941, 48, 330.

37. Downes, F.P.; Ito,K. Compendium of Methods for the Microbiological Examination ofFood $4^{\text {th }}$ ed, APHA, Washington., 2001.

38. Rieckmann, K.H.; Campbell, G.H.; Sax, L. J.; Mrema, J.E.; Lancet., 1978, 1, 221-223.

39. Peters,W.; Richards, W.H.G.; Handbook of Experimental Pharmacology Springer-Verlag Germany., 1984, 179-200.

40. Trager, W.; Jensen, J.B.; Human Malaria Parasites in Continuous Cult. Sci., 1976, 193, 673-675.

41. Lambros, C.; Vanderberg, J. P. J.; Parasitol., 1979, 65, 418-420.

42. Singh, J. J. S. B. Stain.; A review. Indian J. Malariology., 1956, 10, 117-129.

43. Panjarathinam, R.; Text Book of Medical Parasitology, $2^{\text {nd }}$ Ed., Orient Longman., 2007, 329-331.

44. Abu-Khadra, A.S.; Afify, A.S.; Mohamed, A.; Farag, R.S.; Aboul-Enein, H.Y.; The Open Bioactive Compounds Journal., 2018, 6, 1-10.

45. Kayed, S.F.; Farina, Y.; J. of Saudi Chem. Soc., 2020, 24, 236-243.

46. Bhatra, P.; Sharma, J.; Sharma, R.A,; Singh, Y.; Appl. Org. Chem., 2017, 31, 3639. 